\title{
MVA Tumor-specific Neoantigen Boosting Vaccine MVA-209-FSP
}

National Cancer Institute

\section{Source}

National Cancer Institute. MVA Tumor-specific Neoantigen Boosting Vaccine MVA-209FSP. NCI Thesaurus. Code C162806.

An off-the-shelf neoantigen boosting vaccine comprised of a modified Vaccinia virus Ankara (MVA) encoding tumor-specific neoantigens (TSNAs) derived from the same as of yet undisclosed frameshift peptides (FSPs) targeted by the priming vaccine, great ape adenoviral tumor-specific neoantigen priming vaccine GAd-209-FSP, with potential immunostimulatory and antineoplastic activities. Following intramuscular administration of the priming vaccine GAd-209-FSP, the booster MVA tumor-specific neoantigen boosting vaccine MVA-209-FSP may serve to further expand and improve the phenotyping of antigen-specific T-cells targeted to the tumor cells expressing the TSNAS. 